\title{
The perspectives of media and agenda-setting research in times of Big Data
}

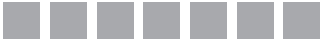 \\ Interview with Prof. Wayne Wanta \\ ORCID: 0000-0002-9337-4006
}

UNIVERSITY OF FLORIDA, USA

DOI: $10.19195 / 1899-5101.11 .2(21) .7$

What is the role of traditional media (such as the printed press) in political communication? Does it still play the role of watchdog?

Traditional media will always play an important role in our societies. Traditional media have two advantages over other media, including social media. First, they have name recognition. I know the New York Times or CNN. I don't necessarily know a social media blogger. So traditional media have a great deal of credibility among the public. Second, they have access to sources and information. A reporter for a traditional medium can request interviews with public officials, or be admitted to news conferences and news events. Social media bloggers can be denied access to news sources. Thus, traditional media can gather news much more efficiently and have a great deal more credibility than non-traditional media. These two advantages point to the importance of traditional media in providing important information to the masses. The importance of the media may be even more significant now because of the false information that fake news websites are producing. For some people, the difference between fake news and real news is unclear.

If you could give researchers some advice on how to conduct research in the hybrid media landscape with the diminishing role of traditional media and enhanced social media, what would be the main suggestion?

Research is certainly different these days. I would point out, though, that a great deal of impact of the media - both social and traditional - is based on selective exposure. Social media have created 'echo chambers', in which members of the public are seeing friends post information that reinforces a person's previously-held positions. This isn't unexpected: people tend to read stories that support their ideas. 
But it could be that traditional media are still setting the public issue agenda. Social media are having a different influence - strengthening their attitudes.

I'm not sure if the role of traditional media has been diminished. Many posts on social media come directly from traditional media. And I know that I trust traditional media more than a post from a non-traditional source. I'm sure other people think that way as well.

An important question in social media research is how a researcher is using social media. If a researcher uses social media as the independent variable, social media essentially would be serving the same function as traditional media. Researchers would be examining if social media content had an impact on the public. On the other hand, if researchers use social media as the dependent variable - as a measure of public opinion - this is very different than research involving traditional media. Previously, researchers tested whether traditional media impacted public attitudes. But the public attitudes were determined by responses to surveys. Now, social media posts are different. The effect being examined is behavioural not attitudinal. People not only become concerned with certain issues, but they become so concerned that they do something - go to the internet and post something about their concern.

\section{We live in an age of Big Data, what is the main suggestion regarding agenda- setting research? What has changed in the intermedia agenda-setting research's process?}

In some ways, Big Data have helped researchers. There are so much data readily available now that the workload for researchers in data collection has been reduced. Survey datasets are available for endless topics. That means that for agenda-setting researchers, half their data have already been collected. All that's left to do is a content analysis of the news media to compare to the survey datasets. Our jobs have become easier.

But our jobs have also become more complicated. Using available datasets means that we do not have control over the questions asked in the surveys. This opens a whole new set of validity issues. Some questions might not be worded as a researcher would have wanted. Or some questions are not appropriate for comparison.

Big Data have also made it easier to do intermedia agenda-setting research. Huge datasets are available for several sources, including sources archiving social media. However, social media are not necessarily the best method to track public attitudes. Dave Weaver, one of the top researchers in our field, once noted that posts to social media may not be showing concern with an issue, as was the focus of the original agenda-setting research. Instead, posts may show an 'interest' - something that is very different than concern. I may post something about crime because I am concerned about this issue, but I may not be interested in the issue. On the 
other hand, I may post something about an upcoming football match because I am interested in it, but I may not be concerned about it. How traditional and social media interact has certainly become more complicated.

Do you personally use any dedicated software that you find useful for your research, especially in respect to the huge amount of digital data?

I really haven't spent too much time testing out new software programs. Some of the content analysis programs have improved a great deal. But most are still most effective finding trends in language but struggle with context.

The big issue with digital data is three-fold. First, researchers have to find ways to access the data - the location and availability. Second, researchers have to find ways to analyze the data - how to put them in some form in order to use statistical measures. Third, researchers have to find how to report what they've found. With the amount of data available, the statistical analyses can be complex.

What media influence the global agenda nowadays? Is it still television, or is it the new media that shape the opinions of their users? Does television still the potential to create the dominating perception and framing of events as the supporters of CNN effect hypothesis would claim?

It's complicated. I'm not sure if there ever was a time when any medium - including CNN - individually had a very powerful impact on the world. There are too many factors that go into public perceptions. There are the biases of members of the public. These impact selective exposure, retention and perception. There are the behaviour patterns of individuals. Some people use multiple media for their news. Others use no media and get exposure to the news only from interactions with other people. There are also influences on the media from governments and public officials. Then there are factors related to the messages. Certain types of reporting can impact different people in different ways. And now with social media, people can influence other people, adding yet another layer of complexity.

The internet and social media have allowed media to transmit their messages across larger geographic areas. CNN continues to reach huge numbers of people through cable television, its website and its social media. But the receivers of those messages have a great deal of power in determining which of those messages are processed. There are so many more options for news information these days.

What is the future of research on agenda-setting? After the theory was supplemented by the $3^{\text {rd }}$ level, in which direction will the theory evolve in the future?

Agenda-setting research has been remarkably resilient through the years. It has withstood hundreds of tests and has continuously evolved. 
My thinking is that the theory will continue to become more and more focused on narrowing the factors related to this process. Originally, researchers looked at one potential influence on the public: media coverage of issues. Different issues influenced people in different ways. Then, researchers looked at a potential influence on the public's perceptions of the issues or objects in the news. This was the second level of agenda setting, or attribute agenda setting. Different attributes of objects in the news influenced different people in different ways. Now we are looking at networks - the third level of agenda setting. Attributes don't appear in isolation. A network of attributes can influence people in different ways.

I think a natural progression would be to examine the context in which these attributes appear. In other words, in first level agenda setting, the analysis looked at the influence of media coverage of an issue/object on the public perception of that issue/object. The second level was concerned with media coverage of attributes linked to objects and the public perception of those attributes and the issue/object. Logically, there should also be a context agenda in which media coverage of the context in which an attribute is linked to an object would influence the public's perception of that context and the public's perception of the attribute and the public's perception of the object. Again, this would be a narrowing of the focus of an analysis.

An example might illustrate this idea. A significant percentage of people in the United States incorrectly believe that former President Barrack Obama is a Muslim. Actually, he's Baptist. Did news coverage influence the perception that he was a Mus$\mathrm{lim}$ ? We did a study examining the context of news coverage linking the Muslim religion to President Obama. We categorized when 'Obama' and 'Muslim' appeared in the same news story. Was the story about Obama meeting a Muslim leader? Or was the story generally about religion? Or was the story about terrorism? We had eight or nine categories, as I recall. We then computed a network analysis that compared the percentage of people claiming Obama was Muslim with the number of news stories in each of these categories. So this study looked at an object (Obama) and an attribute (Muslim religion). Did the linking of the attribute and object among members of the public differ based on the context of this linkage in the news media? I think there is great potential for further research such as this.

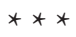

Wayne Wanta, $\mathrm{PhD}$, is a professor in the Department of Journalism at the University of Florida, USA. He is a specialist in political communication and media effects. He has published 200 refereed publications and convention papers. He has lectured and delivered research presentations in 50 different countries. He also has authored or co-authored eight books. His research examines the agenda-setting function of the news media - how news coverage influences the public's perceptions of issues 
and newsmakers. Prof. Wanta has also conducted research in sports journalism, visual communication, Internet use and effects, and negative political advertising. Prof. Wanta has also played an important leadership role in journalism education, having served as the president of the Association for Education in Journalism and Mass Communication.

Before entering the academic field, Wayne Wanta worked for eight years at several newspapers, including the Dallas Times Herald, Austin American-Statesman, Albuquerque Journal, Charleston (S.C.) Post Courier and Wisconsin State Journal. https://www.jou.ufl.edu/staff/wayne-wanta/

Prof. Wayne Wanta was interviewed by Bartłomiej Łódzki, University of Wrocław, and Justyna Arendarska, University of Wrocław, October 16 ${ }^{\text {th }}, 2017$ 